\title{
Comparison of the biometric measurements calculated with Zeiss IOL-Master and WaveLight OB-820
}

This article was published in the following Dove Press journal:

Clinical Ophthalmology

2I April 2017

Number of times this article has been viewed

\author{
Georgios Labiris ${ }^{1,2}$ \\ Panagiota Ntonti ${ }^{2}$ \\ Konstantinos Ntitsos ${ }^{2}$ \\ Andreas Katsanos ${ }^{2}$ \\ Haris Sideroudi ${ }^{2}$ \\ Vasilios Kozobolis ${ }^{1,2}$ \\ 'Department of Ophthalmology, \\ University Hospital of \\ Alexandroupolis, ${ }^{2}$ Eye Institute of \\ Thrace, Alexandroupolis, Greece
}

Correspondence: Georgios Labiris

Department of Ophthalmology,

University Hospital of Alexandroupolis,

68100 Dragana, Alexandroupolis, Greece

Tel +302551030990

Email labiris@usa.net
Background: The primary objective of this study was to investigate the level of agreement between IOL-Master and OB-820 ocular biometers.

Materials and methods: In this prospective randomized case series, we measured the anterior chamber depth (ACD), the axial length (AL), the corneal radii (R1, R2), the ratio of mean corneal radius and spherical equivalent and the corneal astigmatism (cylinder [Cyl]) before and after cataract extraction surgery.

Results: Significant differences between pre- and postoperative data were observed for ACD for both biometric devices $(P<0.01)$ and Cyl parameter in IOL-Master. Range and $95 \%$ limits of agreement (LoA) were clinically significantly different for AL parameter pre- and postoperatively and for R2 and radius and spherical equivalent postoperatively $(P<0.001)$. The rest of the parameters presented sufficient $95 \%$ LoA, which imply good agreement.

Conclusion: In clinical practice, the IOL-master and OB- 820 should not be used interchangeably due to discrepancy in the important AL parameter. Both biometers may provide consistent results regarding $\mathrm{Cyl}, \mathrm{ACD}$ and $\mathrm{R} 1$.

Keywords: cataract, IOL-Master, OB-820, biometry, level of agreement

\section{Introduction}

Ocular biometry is an essential procedure in ophthalmology as it is used to calculate the optical power of the intraocular lens (IOL) that replaces the cataract lens in pseudophakic cataract corrections. ${ }^{1,2}$ An integrated component of optical biometry is accurate measurement of the axial length (AL) of the eye, the most influencing parameter in modern formulas. ${ }^{3,4} \mathrm{AL}$ measurement is based on the principles of transmission, reflection and absorption of light and sound through various media.

The following methods depend on echos in order to get information about the structures inside the eye. ${ }^{5}$ In A-scan biometry (amplitude modulation), the reflection of a thin parallel sound beam is translated by the biometer into spikes arising from the baseline. ${ }^{6}$ The height of the spikes is relevant to the strength of the reflecting echo that strikes each interface in the eye, and the distance between the spikes is relevant to the time needed in order for the echo to travel between each interface. Taking into account the different velocities of the echo transmission in the different ocular media through clear lens $1,640 \mathrm{~m} / \mathrm{s}$, cataract lens $1,629 \mathrm{~m} / \mathrm{s}$ and aqueous $1,532 \mathrm{~m} / \mathrm{s}$, it is possible to differentiate between the different intraocular tissues.

In B-scan biometry, the reflected echoes are represented as a multitude of dots in a gray scale depending on the density of the tissue and are being imaged as a "slice" of 
the depicted structure. B-scan is primarily used to reconstruct ocular topography. ${ }^{7-9}$

However, A-scan ocular biometry suffers from high variability of measurements, which can partly be explained by the fact that the probe has to be in contact with the corneal epithelium. Therefore, a series of ocular biometry technologies have been developed to address the limitations of A-scan biometry: 1) the optical biometry with ZEISS IOL-Master (Carl Zeiss Meditec, Jena, Germany). This is a noncontact biometry device that can determine the $\mathrm{AL}$ of the eye by partial coherence interferometry (PCI). It can also measure the anterior chamber depth (ACD) using visual pachymetry and at the same time provide keratometric data. The AL is measured with an accuracy of $\pm 0.01 \mathrm{~mm}$ in a measurement scale of 14-39 mm. ${ }^{10}$ 2) WaveLight OB-820 (WaveLight, Erlangen, Germany), which is also a noncontact PCI biometry device and the first to use low coherence reflectometry. It provides measurements of the AL, central corneal thickness, ACD and crystalloid lens thickness. It has the ability to analyze 16 different measurements alongside the optical axis and four different keratometric ones with a single scan of the eye. ${ }^{11}$

The function of both these devices is based on Mickelson's interferometer. It is a device that produces interference fringes by splitting a beam of monochromatic light so that one beam strikes a fixed mirror and the other a movable mirror. When the reflected beams are brought back together, an interference pattern results. ${ }^{12}$ This pattern is detected by an appropriate detector. The spatial frequency of the wave crest depends on the difference of the optical route of the two beams until they cohere. In this way, if their difference is an integer multiple of the wavelength $\lambda$, then constructive interference occurs. In case the difference is an integer multiple of $\lambda / 2$, there is destructive interference. Therefore, by altering the distance between the two split beams, the spatial frequency of the waves is also modified. This is accomplished using a reflector that can move forward and backward from the beam splitter. ${ }^{13}$

It becomes obvious that both aforementioned devices utilize cutting-edge technology to calculate certain indexes that are essential in modern cataract surgery. However, a thorough search of the published literature returned no information of the level of agreement between these devices.

Within this context, the primary objective of this study was to assess the level of agreement of a series of biometric parameters between IOL-Master and WaveLight OB-820 in a sample of cataract patients.

\section{Materials and methods Setting}

This was a prospective study. The protocol adhered to the tenets of the Declaration of Helsinki. The institutional review board of the Democritus University of Thrace approved the protocol, and written informed consent was obtained from all participants.

\section{Participants}

Participants were recruited from the Cataract Service on a consecutive-if-eligible basis and underwent full biometric examination using the IOL-Master and OB-820. They were between the ages of 60 and 80 years with senile grade II and grade III cataracts. Exclusion criteria for all study participants included diabetic macular edema, age-related macular degeneration, neurologic disorders that affect the neuroretina and the optic nerve, fundus photocoagulation or other intraocular procedures, edema or scarring of the cornea. Patients with intraoperative complications such as posterior capsule rupture, malposition of IOL, hemorrhage or use of stitches at the corneal incision were also excluded.

\section{Data collection}

The variables for this survey were obtained with IOL-Master and OB-820.

Data collection was performed in a consistent way by the same experienced operator. Regarding IOL-Master, five measurements were obtained for AL calculation with signalto-noise ratio (SNR) at least $>100$. Preoperative data collection was obtained with the appropriate "phakic" setting on the software of the device. On the other hand, postoperative data were collected with the "pseudophakic" setting, which also offers further parameters to determine the type of the IOL, such as silicone, memory and acrylic.

Regarding OB-820, for corneal thickness, 16 point measurements in each eye were arranged in two rings. The outer ring diameter is $2.30 \mathrm{~mm}$, and the inner ring diameter is $1.65 \mathrm{~mm}$. The white-to-white distance is determined by photographing the iris and combining it with the keratometric values. These measurements represent the diameter of an ideal circle. The AL is measured by means of the patient's visual optical line. An integrated conversion factor uses the established IOL calculation formulas that are originally derived from ultrasound biometry. The pupil diameter is obtained after at least five valid measurements. Those are possible even after refractive or other surgical procedures, as there are appropriate settings as well (phakic, aphakic, pseudophakic and silicon-filled eye). 
The following parameters were calculated and evaluated both preoperatively and postoperatively: 1) ACD, 2) AL, 3) horizontal corneal diameter (R1), 4) vertical corneal diameter (R2), 5) radius/spherical equivalent (RSE) and 6) cylinder (Cyl).

\section{Statistical analysis}

The normality of measured data was evaluated by Kolmogorov-Smirnov test. Normal distribution data were assessed by Student's $t$-test. Nonparametric data were assessed with Mann-Whitney $U$-test. Level of agreement between the two devices was assessed by Bland-Altman analysis. $P$-values $<0.05$ were considered as statistically significant. All statistical analyses were performed using SPSS version 16 (SPSS Inc., Chicago, IL, USA).

\section{Results}

Fifty-six patients (28 men and 28 women, $65.2 \pm 11.5$ years) were recruited and populated the study group. Detailed demographic parameters are presented in Table 1. No difficulty in accurate measurements was encountered for our patients' sample. Regarding preoperative and postoperative comparisons for both modalities, nonsignificant differences could be detected for the majority of measured parameters (Table 2). As it was expected, ACD was postoperatively increased both in IOL-Master $(3.17 \pm 0.44 \mathrm{~mm}, 4.53 \pm 0.54 \mathrm{~mm}, P=0.03)$ and in OB-820 (3.23 $\pm 0.41 \mathrm{~mm}, 4.62 \pm 0.34 \mathrm{~mm}, P=0.04)$. However, Cyl demonstrated significant postoperative difference only in IOL-Master $(P=0.02)$. Another important outcome of the study was that, despite the fact that the AL did not significantly change prior to surgery and postoperatively when the parameter was statistically evaluated for each instrument, the actual difference of AL between IOL-Master and OB-820 was significant ( $P=0.03$; Table 3$)$. This finding suggested a probable discrepancy in the limits of agreement (LoA) for the AL parameter, which was confirmed by the Bland-Altman analysis that was performed for all studied parameters. As presented in Bland-Altman plots (Figures 1 and 2), AL presented significant different range and 95\% LoA $(P<0.001)$ for both preoperative and postoperative measurements. In fact, OB- 820 presented significant higher values for all measurements, with the observed

Table I Demographic characteristics of study participants

\begin{tabular}{lllll}
\hline Gender & No & Grade II & Grade III & Age, years \\
\hline Male & 28 & 12 & 16 & $59.5 \pm 10.4$ \\
Female & 28 & 15 & 13 & $61.3 \pm 9.5$ \\
\hline
\end{tabular}

Note: Data are presented as mean \pm standard deviation.
Table 2 Preoperative and postoperative values of measured parameters

\begin{tabular}{|c|c|c|c|c|c|}
\hline & \multicolumn{2}{|c|}{ Preoperative } & \multicolumn{2}{|c|}{ Postoperative } & \multirow[t]{2}{*}{$P$-value } \\
\hline & Mean & SD & Mean & SD & \\
\hline \multicolumn{6}{|l|}{ IOL-Master } \\
\hline $\mathrm{ACD}(\mathrm{mm})$ & 3.17 & 0.44 & 4.53 & 0.54 & $0.03 *$ \\
\hline $\mathrm{AL}(\mathrm{mm})$ & 24.19 & 1.24 & 24.13 & 1.23 & 0.13 \\
\hline $\mathrm{RI}(\mathrm{mm})$ & 7.89 & 0.36 & 7.90 & 0.37 & 0.47 \\
\hline $\mathrm{R} 2(\mathrm{~mm})$ & 7.72 & 0.34 & 7.75 & 0.38 & 0.83 \\
\hline RSE & 7.81 & 0.35 & 7.8 & 0.35 & 0.74 \\
\hline Cyl (D) & 0.78 & 0.56 & 0.86 & 0.54 & $0.02 *$ \\
\hline \multicolumn{6}{|l|}{ OB-820 } \\
\hline$A C D$ & 3.23 & $0.4 \mathrm{I}$ & 4.62 & 0.34 & $0.04 *$ \\
\hline $\mathrm{AL}$ & 24.23 & 1.25 & 24.15 & 1.24 & 0.13 \\
\hline RI & 7.88 & 0.37 & 7.89 & 0.36 & 0.63 \\
\hline $\mathrm{R} 2$ & 7.73 & 0.34 & 7.75 & 0.33 & 0.94 \\
\hline RSE & 7.8 & 0.35 & 7.82 & 0.34 & 0.69 \\
\hline Cyl & 0.79 & 0.5 & 0.81 & 0.45 & 0.21 \\
\hline
\end{tabular}

Notes: RI, horizontal corneal diameter; R2, vertical corneal diameter. *Statistical significance.

Abbreviations: SD, standard deviation; $A C D$, anterior chamber depth; $A L$, axial length; RSE, radius/spherical equivalent; Cyl, cylinder.

discrepancy to be more pronounced for longer eyes (ie, with higher ALs). Moreover, the postoperative values of R2 and RSE parameters demonstrated significant discrepancy as well $(P<0.001)$ (Figures 3 and 4).

\section{Discussion}

Cataract is still the primary cause of reversible blindness in the world, and phacoemulsification is the most commonly performed operation in medicine. ${ }^{14}$ Cutting-edge technology

Table 3 Comparisons of preoperative and postoperative differences

\begin{tabular}{llll}
\hline Parameters & Average & SD & P-value \\
\hline $\begin{array}{l}\text { ACD } \\
\text { IOL-Master }\end{array}$ & 1.36 & & 0.19 \\
$\quad$ OB-820 & 1.39 & 0.41 & \\
AL & & 0.45 & \\
$\quad$ IOL-Master & 0.06 & $0.03^{*}$ \\
$\quad$ OB-820 & 0.08 & 0.05 & \\
RI & & 0.03 & \\
IOL-Master & 0.00 & 0.06 & \\
OB-820 & 0.02 & 0.06 & \\
R2 & & & 0.20 \\
IOL-Master & 0.02 & 0.07 & \\
OB-820 & 0.01 & 0.09 & \\
RSE & & & 0.15 \\
IOL-Master & 0.01 & 0.04 & \\
$\quad$ OB-820 & 0.02 & 0.07 & \\
Cyl & & & 0.79 \\
IOL-Master & 0.08 & 0.51 & \\
OB-820 & 0.02 & 0.50 & \\
\hline
\end{tabular}

Notes: RI, horizontal corneal diameter; R2, vertical corneal diameter. *Statistical significance.

Abbreviations: SD, standard deviation; ACD, anterior chamber depth; AL, axial length; RSE, radius/spherical equivalent; Cyl, cylinder. 


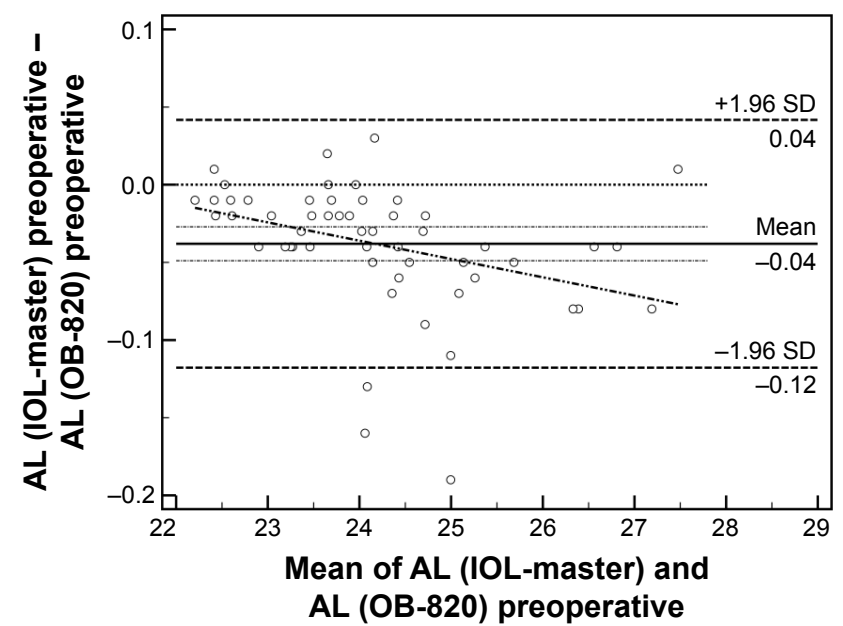

Figure I Bland-Altman plot of AL (preoperative).

Abbreviations: AL, axial length; SD, standard deviation.

in cataract extraction surgery in terms of premium lenses, viscoelastics and emulsification strategies attempts to deliver optimal visual outcomes to the patient. ${ }^{15-17}$ However, among the most essential prerequisites is the accurate calculation of the dioptric power of the IOL that will substitute the cataract lens. ${ }^{18,19}$ A series of mathematical formulas have been developed that predict the necessary dioptric power of the artificial lens taking into account anatomical landmarks of the eye. ${ }^{20-24}$ However, modern ocular biometry presents variability before and after the cataract extraction surgery; moreover, significant discrepancies might be encountered between two different devices. ${ }^{25}$ Moreover, there were several studies that presented the high validity and repeatability of IOL-Master-derived measurements. ${ }^{26,27}$ Nevertheless, none of them presented the validity or repeatability of OB-820 and the comparison of two modalities.

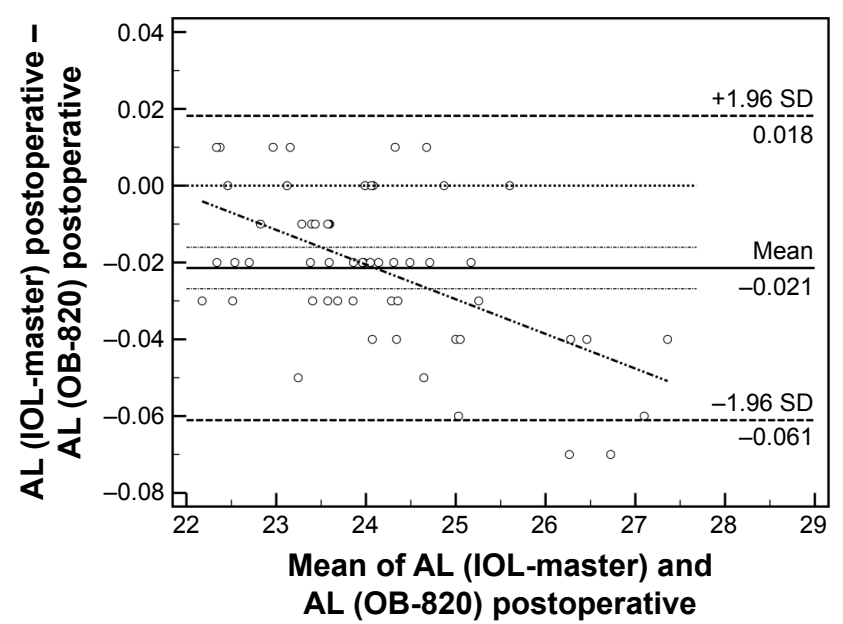

Figure 2 Bland-Altman plot of AL (postoperative). Abbreviations: AL, axial length; SD, standard deviation.

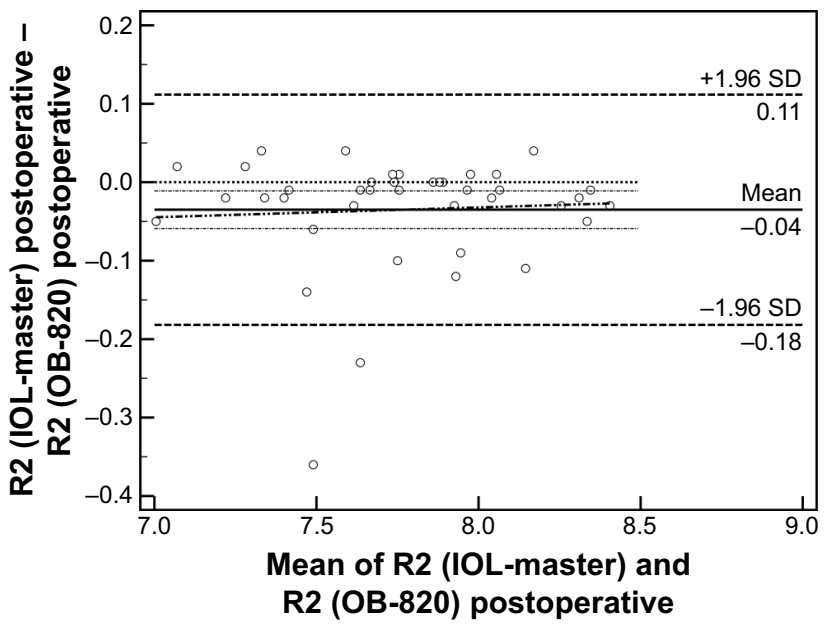

Figure 3 Bland-Altman plot of R2 (postoperative).

Note: R2, vertical corneal diameter.

Abbreviation: SD, standard deviation.

Within this context, this study attempted to evaluate the level of agreement between two prevalent biometric devices (ie, the IOL-Master and OB-820) prior to and following the operation. For this purpose, we recruited a sample of patients who were candidates for cataract extraction surgery, and we measured a series of parameters necessary for the calculation of the IOL power. Our outcomes suggested the following: 1) Further to the expected change in the ACD, both devices demonstrated nonsignificant differences between preoperative and postoperative values for the majority of studied parameters. Only the Cyl parameter presented significant change when measured by the IOL-Master. 2) Significant discrepancy in the level of agreement was detected for the AL both preoperatively and postoperatively, and 3) the postoperative values of R2 and RSE parameters demonstrated

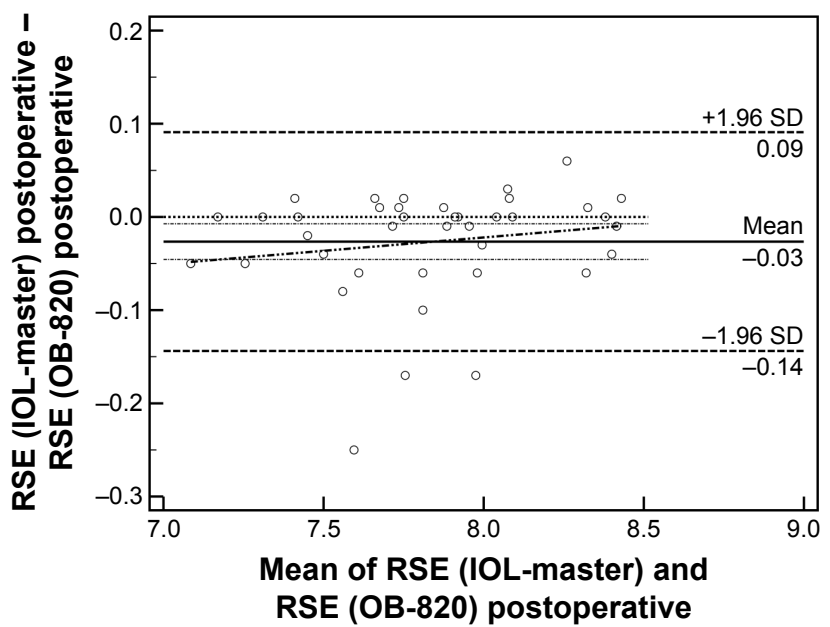

Figure 4 Bland-Altman plot of RSE (postoperative). Abbreviations: RSE, radius/spherical equivalent; SD, standard deviation. 
significant discrepancy as well. Since this is the first study to evaluate the level of agreement between IOL-Master and OB-820, we conducted a thorough literature regarding potential discrepancies in prevalent biometric devices. The majority of published reports are comparative trials regarding IOL-Master and LENSTAR LS 900 (Haag Streit AG). ${ }^{28-30}$ These reports suggest minor differences in measured parameters and adequate level of agreement between the two prevalent instruments. ${ }^{31,32}$

\section{Conclusion}

Our outcomes suggest that OB-820 presents significant difference with IOL-Master in AL measurements, suggesting that these biometry devices should not have been used interchangeably, since it is well known that AL is an integral component of modern formulas that estimate the dioptric power of the IOL. However, larger cohorts of cataractic patients and stratification according to their cataract level are necessary to confirm our results and contribute to the body of knowledge on the important issue of ocular biometry.

\section{Acknowledgment}

No financial support was received for this study.

\section{Disclosure}

None of the authors have received reimbursements, fees, funding, or salary in the past 5 years from an organization that may in any way gain or lose financially from the publication of this manuscript, either now or in the future. None of the authors hold any stocks or shares in an organization that may in any way gain or lose financially from the publication of this manuscript, either now or in the future. None of the authors have received reimbursements, fees, funding, or salary from an organization that holds or has applied for patents relating to the content of the manuscript. None of the authors of this manuscript have a financial interest in any material or method related to this work. No conflicting relationship exists for the authors. None of the authors has any proprietary interests or conflicts of interest related to this submission. It is not simultaneously being considered for publication in any other journal. The authors report no other conflicts of interest in this work.

\section{References}

1. Fedorov SN, Kolinko AI, Kolinko AI. Estimation of optical power of the intraocular lens. Vestn Oftalmol. 1967;80:27-31.

2. Howes FW. Patient workup for cataract surgery. In: Yanoff M, Duker J, editors. Ophthalmology. 4th ed. (Chap. 5.3). St. Louis, MO: Mosby Elsevier; 2009:410-420.
3. Lee AC, Qazi MA, Pepose JS. Biometry and intraocular lens power calculation. Curr Opin Ophthalmol. 2008;19(1):13-17.

4. Wang L, Shirayama M, Ma XJ, Kohnen T, Koch DD. Optimizing intraocular lens power calculations in eyes with axial lengths above 25.0 mm. J Cataract Refract Surg. 2011;37(11):2018-2027.

5. Byrne SF, Green RL. Ultrasound of the Eye and Orbit. 2nd ed. St. Louis, MO: Mosby, Inc; 2002.

6. Byrne SF. A-Scan Axial Eye Length Measurements; a Handbook for IOL Calculations. Mars Hill, NC: Grove Park Publishers; 1995:62-64.

7. Agarwal A, Fan S, Invernizzi A, et al. Characterization of retinal structure and diagnosis of peripheral acquired retinoschisis using high-resolution ultrasound B-scan. Graefes Arch Clin Exp Ophthalmol. 2016;254(1):69-75.

8. Singh R, Invernizzi A, Agarwal A, Kumari N, Gupta A. Enhanced depth imaging spectral domain optical coherence tomography versus ultrasonography B-Scan for measuring retinochoroidal thickness in normal eyes. Retina. 2015;35(2):250-256.

9. Müller HR. The diagnosis of internal carotid artery occlusion by directional Doppler sonography of the ophthalmic artery. Neurology. 1972; 22(8):816.

10. Freeman G, Pesudovs K. The impact of cataract severity on measurement acquisition with the IOLMaster. Acta Ophthalmol Scand. 2005; 83(4):439-442.

11. Buckhurst PJ, Wolffsohn JS, Shah S, Naroo SA, Davies LN, Berrow EJ. A new optical low coherence reflectometry device for ocular biometry in cataract patients. Br J Ophthalmol. 2009;93(7):949-953.

12. Hariharan P. Basics of Interferometry. San Diego, CA: Academic Press; 2010.

13. Kanellopoulos AJ, Asimellis G. Correlation between central corneal thickness, anterior chamber depth, and corneal keratometry as measured by Oculyzer II and WaveLight OB820 in preoperative cataract surgery patients. J Refract Surg. 2012;28(12):895-900.

14. WHO. Global Data on Visual Impairments 2010. Geneva: WHO; 2012:6.

15. Labiris G, Giarmoukakis A, Patsiamanidi M, Papadopoulos Z, Kozobolis VP. Mini-monovision versus multifocal intraocular lens implantation. J Cataract Refract Surg. 2015;41(1):53-57.

16. Labiris G, Sideroudi H, Rousopoulos K, Kozobolis VP. Cohesive versus dispersive-cohesive ophthalmic viscosurgical device in torsional intelligent phaco. J Cataract Refract Surg. 2015;41(3):681-682.

17. Roberts TV, Lawless M, Sutton G, Hodge C. Update and clinical utility of the LenSx femtosecond laser in cataract surgery. Clin Ophthalmol. 2016;10:2021.

18. Bollinger KE, Langston RHS. What can patients expect from cataract surgery? Cleve Clin J Med. 2008;75(3):193.

19. Schein OD, Katz J, Bass EB, et al. The value of routine preoperative medical testing before cataract surgery. $N$ Engl J Med. 2000;342(3): $168-175$.

20. Holladay JT. Standardizing constants for ultrasonic biometry, keratometry, and intraocular lens power calculations. J Cataract Refract Surg. 1997;23(9):1356-1370.

21. Ng DT, Rowe NA, Francis IC, et al. Intraoperative complications of 1000 phacoemulsification procedures: a prospective study. $J$ Cataract Refract Surg. 1998;24(10):1390-1395.

22. Kurimoto Y, Park M, Sakaue H, Kondo T. Changes in the anterior chamber configuration after small-incision cataract surgery with posterior chamber intraocular lens implantation. Am J Ophthalmol. 1997;124(6):775-780.

23. Pereira FA, Cronemberger S. Ultrasound biomicroscopic study of anterior segment changes after phacoemulsification and foldable intraocular lens implantation. Ophthalmology. 2003;110(9):1799-1806.

24. Long DA, Monica ML. A prospective evaluation of corneal curvature changes with 3.0 - to $3.5-\mathrm{mm}$ corneal tunnel phacoemulsification. Ophthalmology. 1996;103(2):226-232.

25. Rozema JJ, Wouters K, Mathysen DG, Tassignon MJ. Overview of the repeatability, reproducibility, and agreement of the biometry values provided by various ophthalmic devices. Am J Ophthalmol. 2014;158(6): 1111-1120. 
26. Lopez de la Fuente C, Sanchez-Cano A, Segura F, Pinilla I. Comparison of anterior segment measurements obtained by three different devices in healthy eyes. Biomed Res Int. 2014;2014:498080.

27. Dinc UA, Gorgun E, Oncel B, Yenerel MN, Alimgil L. Assessment of anterior chamber depth using Visante optical coherence tomography, slitlamp optical coherence tomography, IOL Master, Pentacam and Orbscan IIz. Ophthalmologica. 2010;224(6):341-346.

28. Rabsilber TM, Jepsen C, Auffarth GU, Holzer MP. Intraocular lens power calculation: clinical comparison of 2 optical biometry devices. $J$ Cataract Refract Surg. 2010;36(2):230-234.

29. Chen Y-A, Hirnschall N, Findl O. Evaluation of 2 new optical biometry devices and comparison with the current gold standard biometer. J Cataract Refract Surg. 2011;37(3):513-517.
30. Hui S, Yi L. Comparison of two optical biometers in intraocular lens power calculation. Indian J Ophthalmol. 2014;62(9):931.

31. Mylonas G, Sacu S, Buehl W, Ritter M, Georgopoulos M, SchmidtErfurth U. Performance of three biometry devices in patients with different grades of age-related cataract. Acta Ophthalmol. 2011;89(3): e237-e241.

32. Shin JW, Seong M, Kang MH, Lee YG. Comparison of ocular biometry and postoperative refraction in cataract patients between Lenstar ${ }^{\circledR}$ and IOL Master ${ }^{\circledR}$. J Korean Ophthalmol Soc. 2012;53(6):833-838.
Clinical Ophthalmology

\section{Publish your work in this journal}

Clinical Ophthalmology is an international, peer-reviewed journal covering all subspecialties within ophthalmology. Key topics include: Optometry; Visual science; Pharmacology and drug therapy in eye diseases; Basic Sciences; Primary and Secondary eye care; Patient Safety and Quality of Care Improvements. This journal is indexed on

\section{Dovepress}

PubMed Central and CAS, and is the official journal of The Society of Clinical Ophthalmology (SCO). The manuscript management system is completely online and includes a very quick and fair peer-review system, which is all easy to use. Visit http://www.dovepress.com/ testimonials.php to read real quotes from published authors. 